\title{
Design challenges of implantable pressure monitoring system
}

\section{Guangqiang Jiang*}

Alfred E. Mann Foundation for Scientific Research, Santa Clarita, CA, USA

\section{Edited by:}

Victor Pikov, Huntington Medical

Research Institutes, USA

Reviewed by:

Ellis Meng,

University of Southern California, USA

Victor Pikov, Huntington Medical

Research Institutes, USA

${ }^{*}$ Correspondence:

Guangqiang Jiang, Alfred E. Mann

Foundation for Scientific Research

25134 Rye Canyon Loop, Santa Clarita,

CA 91354, USA.

e-mail: jiang@aemf.org
Pressure in various organs and body parts, such as blood vessels, heart, brain, eyes, bladder and $\mathrm{Gl}$ tracts, is an important indication of health. Long term, continuous pressure monitoring is critically needed for a number of applications. When combined with existing neuro-prosthetics devices, they may provide better solutions to many neural disorders. First efforts toward a long-term implantable pressure monitoring system were initiated more than 40 years ago. However, a reliable, safe and implantable pressure sensor for long-term applications is not yet commercially available. This paper attempts to reveal the design challenges associated with the development of a long-term implantable pressure sensor.

Keywords: design challenges, implantable pressure sensor, long term, closed-loop neuro-prosthetics devices

\section{INTRODUCTION}

Long-term, continuous pressure monitoring is critically needed for a number of applications, including the monitoring of the intraarterial pressure of patients with hypertension, the intraocular pressure of patients with glaucoma (Schnakenberg et al., 2000), the bladder pressure of patients with neurogenic bladder dysfunction (Sansen et al., 1987; Coosemans and Puers, 2005). More frequent intraocular pressure (IOP) measurements are desirable not only for a better understanding of glaucoma, which is characterized by an elevated IOP, but also for a better management of individual patients (Schnakenberg et al., 2000). Real time, continuous bladder pressure information can be used to stimulate the sphincter muscles in an intelligent way (Sansen et al., 1987). This can be particularly useful for patients with neurogenic bladder dysfunction (Coosemans and Puers, 2005). A shunt system currently used for hydrocephalus or traumatic brain injury treatment could be more effective with a continuous intracranial pressure feedback (Olsen et al., 1967). Currently, the most common methods for continuous pressure measurement are through a catheter-based system. These methods are often only suitable for short term measurement since percutaneous connections through skin present infection risks. Catheter-based systems prohibit free movement of the subject and limit feasibility to clinical settings. An implantable telemetric pressure sensor would overcome these limitations and problems. The combination of microelectronic circuits and wireless technology led to the first efforts in the 1960s and 1970s towards developing implantable pressure sensors (Van Citters et al., 1966; Olsen et al., 1967; Casadei et al., 1972; Cooper and Beale, 1977). However, a reliable, safe and implantable pressure sensor for long term applications is not yet available in the market.

\section{MAJOR PRESSURE SENSING METHODS}

Pressure sensors can vary drastically in technology, design, performance, application suitability and cost. A conservative estimate would be that there may be over 50 technologies and at least 300 companies making pressure sensors worldwide (http://www. absoluteastronomy.com/topics/Pressure_sensor). This paper only discusses two types of pressure sensors that are widely studied for biomedical applications: namely, the piezo-resistive and capacitive. The absolute pressure range for biomedical applications is typically from 400 to $1200 \mathrm{mmHg}$. A piezo-resistive sensor relies on the resistivity changes of the piezo-resistors embedded on the diaphragmatic membrane when it deforms due to pressure change. Capacitive sensing uses the diaphragm deformation-induced capacitance change. Capacitive electrodes are often formed on the top and bottom surfaces of the sealed cavity and to convert the information about pressure into an electrical signal (Varadan et al., 2001). Both types of sensors can be fabricated in a miniaturized fashion thanks to the advancement of micro-electro-mechanical systems (MEMS) technology. Piezo-resistive sensors are normally used in catheter based pressure sensing systems. They may be better suited for periodic pressure measurement rather than for dynamic telemetric applications as the power consumption is high. Piezoresistive sensor often produces a large temperature error (Flick and Orglmeister, 2000). Capacitive sensors are more suited to implantable devices because of their sensitivity to pressure changes, low noise and low temperature sensitivity (Akar et al., 2001). Most importantly, the reduced power consumption is essential for wireless applications as power is not randomly available (Puers, 1993). For this reason research in the area of implantable pressure sensors tends to focus on the use of capacitive MEMS devices (Sansen et al., 1987; Chiang et al., 2007; Ginggen et al., 2008). Drawbacks of a capacitive sensor for implantable applications include the need for active implanted electronics and its instability over various environmental conditions.

\section{DESIGN CHALLENGES OF AN IMPLANTABLE PRESSURE MONITORING SYSTEM}

An implantable pressure monitoring system should at least consist of two parts: an implantable pressure sensor and an external device. The external device needs to interface with, and possibly provide power and commands to, the implanted sensor, and downloads data from it. For wireless sensing applications, data transfer can be achieved through the use of radio frequency 
(RF), infrared or optical media. Of these options, RF is the most popular. There are several prescribed frequency bands that are available for the medical device community to choose from (Akyildiz et al., 2002; Budinger 2003): the very high frequency (VHF) band at 174-216 MHz, the ultra high frequency (UHF) bands at $401-406 \mathrm{MHz}$ and $450-470 \mathrm{MHz}$, and several narrow bands within the industrial, scientific and medical (ISM) bands of $6765 \mathrm{kHz}$ to $245 \mathrm{GHz}$. Selection of a frequency for wireless implant system communication depends on the location of the implant, power needed to run the implant device, bandwidth needed to download and upload data to and from the implant device, and more. In general, for an implantable device utilizing $\mathrm{RF}$ communication, a frequency in the low- $\mathrm{MHz}$ region provides a good compromise between bandwidth and tissue absorption (DeHennis, 2004; Yu, 2004). The challenges associated with the electronics design for the external device or at system level will be discussed elsewhere. This paper focuses on the challenges associated with the implantable device design.

Besides pressure sensor(s), some electronic circuitry for firststage data processing, power management and signal receiving and transmitting is preferably included in the implantable device. Common requirements for an implantable pressure sensor include: the device must be small, have insignificant drift over the period of its use, be compatible with modern imaging techniques (Computed Tomography (CT) scan, Magnetic Resonance Imaging (MRI) scan and ultrasound, etc.), be hermetic and biocompatible, and comply with the Food and Drug Administration (FDA), American National Standard Institute (ANSI), Association of Medical Instrumentation (AAMI) and International Standard Organization (ISO) guidelines. Geometry, measurement range, data transmission rate, sampling rates, and sensitivity are the other design considerations which strongly depend on the application. The following discussion addresses the most critical of these.

\section{DRIFT}

Drift has been one of the major challenges for realization of a reliable implantable pressure sensor. The drift of a catheter based pressure sensor is handled by frequent re-calibration through an external known reference pressure. However it is impossible to recalibrate a pressure sensor once it is implanted without surgical intervention. Two kinds of drift are offset drift and sensitivity drift. Offset drift refers to the changes in a sensor from its calibrated state. This may be caused by the changes of mechanical properties of the sensing element (diaphragm) due to aging from its calibrated point, or changes of the reference pressure cavity (absolute type) because gases leak into a vacuum cavity or out from the gas-filled cavity. Sensitivity drift may be caused by mechanical fatigue (elastic modulus change) of the diaphragm, or mechanical stiffness changes due to attachment of cells or tissues to the sensing diaphragm over time or diaphragm material corrosion. Zhou et al. (2000) reported silicon wafer showed sign of corrosion in Bicarbonate Buffered Saline (BBS) at $37^{\circ} \mathrm{C}$ after about 6 months. The properties of the materials used for constructing the pressure sensor are often temperature dependent, which determines the temperature dependency of both offset drift and sensitivity drift. MEMS sensor designers developed intelligent circuits to compensate for both offset drift and sensitivity drift of their specific sensor products. Packaging of the pressure sensor can also lead to both sensitivity and offset drift. This impact may be reduced through careful design of device packaging and materials selection.

One way to minimize the drift induced by the attachment of various biological substances to the diaphragm is to design the mechanical stiffness of the diaphragm much greater than the mechanical stiffness of the potential attached tissues. However, this will significantly reduce sensor sensitivity. Frischholz et al. (2007) proposed a biomaterial nano-layer to reduce cell growth on the sensing element. However, no detail about the method or materials to be used was offered in his paper. One other way is to protect the sensing element from direct contact with connective tissue by containing the pressure sensor in a bellow structure filled with silicone oil or other uncompressible liquid. One unavoidable challenge of this approach is guaranteeing the external pressure is transmitted to the sensing element freely without distortion. Another challenge is compensating for the offset drift induced by the temperature dependency of the coefficient of thermal expansion of the uncompressible fluid. A silicone pearl was used by Flick and Orglmeister (2000) as the transmitting media to measure pressure indirectly where a silicone capsule is responsible for the protection of the system. No discussion on the above mentioned challenges is offered in this paper.

\section{DATA SAMPLING RATE AND LOW POWER CONSUMPTION}

Sampling rate is a variable that depends on the application. Higher sampling rate is preferred since it often provides more information. However, the power consumption increase of the telemetry commensurates with the increase in the data transmission sampling rate. For a battery powered device, higher power consumption means shorter lifetime of the device. For a remotely powered device, higher power consumption will decrease its communication distance. Moreover, more heat will be generated due to higher power consumption from the implant which may damage the contact tissue. ISO 14708 - Clause 17 requires any part of an implantable device surface temperature increase should be less than $2^{\circ}$ Kelvin. The implementation of micro-fabrication technologies has allowed the development of devices with decreased size and power consumption.

\section{HERMETIC AND BIOCOMPATIBLE PACKAGE}

The development of a durable packaging solution for a sensor that is compatible and resistant to the human fluids is a well known challenge. One such factor that makes it so challenging is the corrosive environment in which the implant device has to survive (Kanda and Aoshinma, 1981). Another factor is the biocompatibility and hemo-compatibility of the package material (Yuen et al., 1990). It is crucial for the life of a device and the protection of the body to use a material that would satisfy both needs. Kotzar et al. (2002) evaluated the biocompatibility of several typical MEMS materials, including $\mathrm{Si}$, poly-Si, $\mathrm{SiO}_{2}, \mathrm{Si}_{3} \mathrm{~N}_{4}, \mathrm{SiC}$, Ti and $\mathrm{SU}-8$ epoxy photoresist and reported negative biocompatibility issues. Other biocompatible materials that have been successfully used for implantable medical device packaging include titanium and its alloys, noble metals and their alloys, biograde stainless steels, some cobalt-based alloys, tantalum, niobium, titanium-niobium alloys, Nitinol (a shape memory alloy), MP35N ${ }^{\circledR}$ (a nickel-cobalt-chromium-molybdenum alloy), alumina, zirconia, and some biocompatible glasses and polymers (Jiang and Zhou, 2009). 
A hermetic package is necessary to prevent moisture from ingression into the implant device that might cause failures such as open circuits, surface electrical leakage, and electrical shorts due to moisture-promoted dendritic growth of silver and gold. Ingress of other active gases, such as oxygen, could also cause attachment failure of solder-attached components due to solder oxidization.

One particular challenge associated with packaging implantable pressure sensors comes from the fact that MEMS pressure sensor performance is very sensitive to packaging-induced stress and the packaging processes. The two most typical materials used for MEMS pressure sensor manufacturing are silicon and glass. Corning 7740 $\left(\right.$ Pyrex $\left.^{\circledR}\right)$, Corning 7070, Corning 1729, Corning 9626, Schott 8330 (Tempax ${ }^{\circledast}$ ) and SD-2 (HOYA) are the most popular glass materials because they have a close coefficient of thermal expansion (CTE) to silicon (Rogers and Kowal, 1995; Harz and Engelke, 1996). Anodic bonding is widely used to form the hermetic seal between glass and silicon. At the present time, most silicon micro-sensors are made using this technology. It does not need interlayer materials, which simplifies the manufacturing process and eliminates biocompatibility concerns. Good biocompatibility of both Si and Pyrex ${ }^{\circledast}$ glass has been documented (Dokmeci et al., 1997; Kotzar et al., 2002).

Similar to micro pressure sensor manufacturing, minimizing residual stress is the most important goal for packaging pressure sensors for implantable applications. If direct bonding to the pressure sensor body is required, selecting materials that have wellmatched CTEs for pressure sensor packaging is the key. Minimizing parasitic capacitance should always be kept in mind when designing a package for a capacitive pressure sensor. Increased parasitic capacitance will degrade the pressure sensitivity of the implantable pressure device. Parasitic capacitance could be reduced by using short leads, enlarging distance between leads and low dielectric constant materials between leads.

\section{REFERENCES}

Akar, O., Akin, T., and Najafi, K. (2001). A wireless batch sealed absolute capacitive pressure sensor. Sens. Actuators A. Phys. 95, 29-38.

Akyildiz,I.F.,Su,W.,Sankarasubramaniam, Y., and Cayirci, E. (2002). Wireless sensor networks: a survey. Comput. Networks 38, 393-422.

Budinger, T. F. (2003). Biomonitoring with wireless communications. Annu. Rev. Biomed. Eng. 5, 383-412.

Casadei, F. W., Gerold, M., and Baldinger, E. (1972). Implantable blood pressure telemetry system. IEEE Trans. Biomed. Eng. BME-9, 334-341.

Chiang, C. C., CLin, C. K., and Ju, M. S. (2007). An implantable capacitive pressure sensor for biomedical applications. Sens. Actuators A. Phys. 134 382-388.

Cooper, R., and Beale, D. (1977). Radio telemetry of intraocular pressure in vitro. Invest. Ophthalmol. Vis. Sci. 16, $168-171$.

Coosemans, J., and Puers, R. (2005). An autonomous bladder pressure

\section{OTHER DESIGN CHALLENGES}

One other design consideration for the implantable device is its compatibility with popular imaging diagnostic tools, such as CT scan, MRI and ultrasound. MRI is a tool that is especially useful in neurological (brain), musculoskeletal, cardiovascular, and oncological (cancer) imaging. The implantable device has to be designed so it is at least MR safe if not MR compatible. Ferrite material can significantly improve RF communication efficiency. However, a second thought must be given when one is to use ferrite materials in the implant device since it interacts with MR and cause heating and significant imaging distortion. Overdose X-ray exposure could permanently erase the stored data in memory chips and potentially cause device failure, so cautions are to be given. High intensity ultrasound energy could potentially damage electronic assemblies inside the implant and the package and cause device malfunction or failure, which raises the bar of requirements for both electronics assembly and package integrity.

\section{CONCLUSION}

Long term, continuous pressure monitoring is critically needed for a number of applications. An implantable telemetric pressure monitoring system will fill this important need. Minimal drift, adequate data transmission and sampling rates while maintaining low power consumption, and biocompatible and hermetic package are among the top design challenges. Above challenges must be overcome before a reliable, long term, and continuous pressure monitoring system can be realized.

\section{ACKNOWLEDGMENTS}

The authors would like to thank Dr. G. Schnittgrund and S. Grannis for their detailed review of the manuscript. Technical discussion with S. Schmidt is very helpful. monitoring system. Sens. Actuators A Phys. 123-124, 155-161.

DeHennis, A. D. (2004). RemotelyPowered Wireless Monitoring Systems. Ph.D. Dissertation, University of Michigan, Ann Arbor.

Dokmeci, M. R., Von Arx, J.A., and Najafi, K. (1997). Accelerated testing of anodically bonded glass-silicon packages in salt water. In Proceedings of 9th International Conference on Solid State Sensors and Actuators, Transducers '97, Chicago, pp. 283-286.

Flick, B. B., and Orglmeister, R. (2000). A portable microsystem-based telemetric pressure and temperature measurement unit. IEEE Trans. Biomed. Eng. 47, 12-16.

Frischholz, M., Sarmento, L., Wenzel, M., Aquilina, K., Edwards, R., and Coakham, H. B. (2007). Telemetric implantable pressure sensor for short-term and long-term monitoring of intracranial pressure, 514. In Proceedings of the 29th Annual International Conference of the IEEE EMBS, Lyon, France, August 23-26.
Ginggen, A., Tardy, Y., Crivelli, R., Bork, T., and Renaud, P. (2008). A telemetric pressure sensor system for biomedical applications. IEEE Trans. Biomed. Eng. $55,1374-1381$.

Harz,M., and Engelke,H.(1996). Curvature changing or flateening of anodically bnded silicon and borosilicate glass. Sens. Actuators A. Phys. 55, 201-209.

Jiang, G., and Zhou, D. (2009). Technology Advances and Challenges in Hermetic Packaging for Implantable Medical Devices. In Implantable Neural Prostheses 2 - Techniques and Engineering Approaches, Biological and Medical Physics, Biomedical Engineering. D. D. Zhou and E. Greenbaum,eds. (NewYork, Springer). pp. 27-61.

Kanda, Y., and Aoshima, R., Takada, A. (1981). Blood compatibility of components and materials in silicon integrated circuits. Electron Lett. 17, 558-559.

Kotzar, G., Freas, M., Abel, P., Fleischman, A., Roy, S., Zorman, C., Moran, J.M., and Melzak, J. (2002). Evaluation of MEMS materials of construction for implantable medical devices. Biomaterials 23, 2737-2750.

Olsen, E. R., Collins, C. C., Loughborough, W. F., Richards, V., Adams, J. E., and Pinto, D. W. (1967). Intracranial pressure measurement with a miniature passive implanted pressure transensor. Am. J. Surg. 113, 727-729.

Puers, R. (1993). Capacitive sensors: when and how to use them. Sens. Actuator A. Phys. 37/38, 93-105.

Rogers, T., and Kowal, J. (1995). Selection of glass, anodic bonding conditions and material compatibility for siliconglass capacitive sensors. Sens. Actuators A. Phys. 46-47, 113-120.

Sansen, W., Vereecken, R., Puers, R., Folens, G., and Van Nuland, T. (1987). A closed loop system to control the bladder function. In Proceedings of the Ninth Annual Conference of the IEEE Engineering in Medicine and Biology Society, Boston, November 13-16, pp. 1149-1150.

Schnakenberg, U., Walter, P., Bogel, G. V., Kruger, C., Ludtke-Handjery, H. C., Richter, H. A., Specht, W., Ruokonen, 
P., and Mokwa, W. (2000). Initial investigations on systems for measuring intraocular pressure. Sens. Actuators 85, 287-291.

Van Citters, R. L., Kemper, W. S., and Franklin, D. L. (1966). Blood pressure responses of wild giraffes studied by radio telemetry. Science 152 , 384-386.

Varadan, V. K., Jiang, X., and Varadan, V. (2001). Microstereolithography other fabrication techniques for 3D MEMS. Chichester, John Wiley \& Sons Ltd. p. 260.
Yu, H. (2004). A Wireless Microsystem for Multichannel Neural Recording Microprobes. Ph.D. Dissertation, University of Michigan, Ann Arbor.

Yuen, T., Agnew, W., Bullara, L., and McCreery,D.B.(1990).Biocompatibility of electrodes and materials in the central nervous system. In Neural Prostheses: Fundamental Studies, W. Agnew, and D.McCreery, eds (Englewood Cliffs, NJ, Prentice Hall), pp 171-321.

Zhou, D., Mech, B., and Greenberg, R. (2000). Accelerated corrosion tests on Silicon wafers for implantable medical devices. Proceedings of 198th Electrochemical Society Meeting, Phoenix, p. 363.

Conflict of Interest Statement: The author declares that the research was conducted in the absence of any commercial or financial relationships that could be construed as a potential conflict of interest.

Received: 12 January 2010; paper pending published: 13 January 2010; accepted: 08 February 2010; published online: 26 February 2010.
Citation: Jiang G (2010) Design challenges of implantable pressure monitoring system. Front. Neurosci. 4:2. doi: 10.3389/neuro.20.002.2010

This article was submitted to Frontiers in Neuroprosthetics, a specialty of Frontiers in Neuroscience.

Copyright (๑) 2010 Jiang. This is an openaccess article subject to an exclusive license agreement between the authors and the Frontiers Research Foundation, which permits unrestricted use, distribution, and reproduction in any medium, provided the original authors and source are credited. 